\title{
Visa Processing Information System
}

\author{
M.Kamakshi Karthiga ${ }^{1}$, Mrs.S.Nithya ${ }^{2}$ \\ ${ }^{I}$ (Research Scholar, Department of Computer Science \& Engineering, PRIST University, Tamilnadu, India.) \\ ${ }_{2}^{2}$ (Assistant Professor, Department of Computer Science \& Engineering, PRIST University, Tamilnadu, India.)
}

\begin{abstract}
Visa Resource - a Very important Department in any Organization. Our Project gives the depth solution to one of its important requirements as Visa Data Processing. The client of this project is any organization who is having many abroad clients especially US clients. This type of organizations is frequently sending their employees for their overseas clients. Our project is used to process their visa and store all the details regarding the passport holder. Our project will handle all type of visas, and very much concentrate in visa processing. It will generate all possible reports, which are need by Consulate. It is a Client Server Project, Administrator can be able to create many no of users and the users are called as Customers. Administrator will describe the utilities accessed by an Customer
\end{abstract}

Keywords: Active server pages extensible file, Common language runtime, Internet information services, Just in time, Software specification,

\section{Introduction}

HR This web-based application is for the employees of an organization for processing their visa applications. Manager is considering as Site Admin and he is having the control over all the users. Administrator can create any number of Customer logins and authenticated them. Hr Executives are the responsible persons to execute the process of Visa applications and their Onsite Details. Companies Employees, those who are willing enquire about visa status are able to login into this portal. Their empID is considered as username and their password will be given by Company HR during his/her joining. Onsite employees may apply for visa renewal through this project.

\section{Microsoft .Net Framework}

\section{Literature Survey}

The .NET Framework is a new computing platform that simplifies application development in the highly distributed environment of the Internet. The .NET Framework is designed to fulfill the following objectives:

- To provide a code-execution environment that minimizes software deployment and versioning conflicts.

- To provide a code-execution environment that guarantees safe execution of code, including code created by an unknown or semi-trusted third party.

- To provide a code-execution environment that eliminates the performance problems of scripted or interpreted environments.

- To make the developer experience consistent across widely varying types of applications, such as

- Windows-based applications and Web-based applications.

- To provide a consistent object-oriented programming environment whether object code is stored and executed locally, but Internet-distributed, or executed remotely.

- To build all communication on industry standards to ensure that code based on the .NET Framework can integrate with any other code.

The .NET Framework has two main components: The Common Language Runtime and the .NET Framework class library. The Common Language Runtime is the foundation of the .NET Framework. You can think of the runtime as an agent that manages code at execution time, providing core services such as memory management, thread management, and remoting, while also enforcing strict type safety and other forms of code accuracy that ensure security and robustness. In fact, the concept of code management is a fundamental principle of the runtime. Code that targets the runtime is known as managed code, while code that does not target the runtime is known as unmanaged code. The class library, the other main component of the .NET Framework, is a comprehensive, object-oriented collection of reusable types that you can use to develop applications ranging from traditional command-line or graphical user interface (GUI) applications to applications based on the latest innovations provided by ASP.NET, such as Web Forms and XML Web services.The .NET Framework can be hosted by unmanaged components that load the common language runtime into their processes and initiate the execution of managed code, thereby creating a software environment that can exploit both managed and unmanaged features. The .NET Framework not only provides 
several runtime hosts, but also supports the development of third-party runtime hosts.For example, ASP.NET hosts the runtime to provide a scalable, server-side environment for managed code. ASP.NET works directly with the runtime to enable Web Forms applications and XML Web services, both of which are discussed later in this topic.Internet Explorer is an example of an unmanaged application that hosts the runtime (in the form of a MIME type extension). Using Internet Explorer to host the runtime enables you to embed managed components or Windows Forms controls in HTML documents. Hosting the runtime in this way makes managed mobile code (similar to Microsoft ${ }^{\circledR}$ ActiveX® ${ }^{\circledR}$ controls) possible, but with significant improvements that only managed code can offer, such as semi-trusted execution and secure isolated file storage. The following illustration shows the relationship of the common language runtime and the class library to your applications and to the overall system. The illustration also shows how managed code operates within a larger architecture.

\section{A. Features of the Common Language Runtime:}

The common language runtime manages memory, thread execution, code execution, code safety verification, compilation, and other system services. These features are intrinsic to the managed code that runs on the common language runtime. With regards to security, managed components are awarded varying degrees of trust, depending on a number of factors that include their origin (such as the Internet, enterprise network, or local computer). This means that a managed component might or might not be able to perform file-access operations, registry-access operations, or other sensitive functions, even if it is being used in the same active application.The runtime enforces code access security. For example, users can trust that an executable embedded in a Web page can play an animation on screen or sing a song, but cannot access their personal data, file system, or network. The security features of the runtime thus enable legitimate Internet-deployed software to be exceptionally feature rich.The runtime also enforces code robustness by implementing a strict type- and code-verification infrastructure called the common type system (CTS). The CTS ensures that all managed code is self-describing. The various Microsoft and third-party language compilers Generate managed code that conforms to the CTS. This means that managed code can consume other managed types and instances, while strictly enforcing type fidelity and type safety.In addition, the managed environment of the runtime eliminates many common software issues. For example, the runtime automatically handles object layout and manages references to objects, releasing them when they are no longer being used. This automatic memory management resolves the two most common application errors, memory leaks and invalid memory references. The runtime also accelerates developer productivity. For example, programmers can write applications in their development language of choice, yet take full advantage of the runtime, the class library, and components written in other languages by other developers. Any compiler vendor who chooses to target the runtime can do so. Language compilers that target the .NET Framework make the features of the .NET Framework available to existing code written in that language, greatly easing the migration process for existing applications. While the runtime is designed for the software of the future, it also supports software of today and yesterday. Interoperability between managed and unmanaged code enables developers to continue to use necessary COM components and DLLs.The runtime is designed to enhance performance. Although the common language runtime provides many standard runtime services, managed code is never interpreted. A feature called just-in-time (JIT) compiling enables all managed code to run in the native machine language of the system on which it is executing. Meanwhile, the memory manager removes the possibilities of fragmented memory and increases memory locality-of-reference to further increase performance.Finally, the runtime can be hosted by high-performance, server-side applications, such as Microsoft ${ }^{\circledR}$ SQL Server ${ }^{\mathrm{TM}}$ and Internet Information Services (IIS). This infrastructure enables you to use managed code to write your business logic, while still enjoying the superior performance of the industry's best enterprise servers that support runtime hosting.

\section{B. .NET Framework Class Library:}

The .NET Framework class library is a collection of reusable types that tightly integrate with the common language runtime. The class library is object oriented, providing types from which your own managed code can derive functionality. This not only makes the .NET Framework types easy to use, but also reduces the time associated with learning new features of the .NET Framework. In addition, third-party components can integrate seamlessly with classes in the .NET Framework.For example, the .NET Framework collection classes implement a set of interfaces that you can use to develop your own collection classes. Your collection classes will blend seamlessly with the classes in the .NET Framework. As you would expect from an object-oriented class library, the .NET Framework types enable you to accomplish a range of common programming tasks, including tasks such as string management, data collection, database connectivity, and file access. In addition to these common tasks, the class library includes types that support a variety of specialized development scenarios. For example, you can use the .NET Framework to develop the following types of applications and services:

- Console applications.

- Scripted or hosted applications. Windows GUI applications (Windows Forms). 
- $\quad$ ASP.NET applications.

- XML Web services.

- Windows services.

For example, the Windows Forms classes are a comprehensive set of reusable types that vastly simplify Windows GUI development. If you write an ASP.NET Web Form application, you can use the Web Forms classes.

\section{Client Application Development:}

Client applications are the closest to a traditional style of application in Windows-based programming. These are the types of applications that display windows or forms on the desktop, enabling a user to perform a task. Client applications include applications such as word processors and spreadsheets, as well as custom business applications such as data-entry tools, reporting tools, and so on. Client applications usually employ windows, menus, buttons, and other GUI elements, and they likely access local resources such as the file system and peripherals such as printers. Another kind of client application is the traditional ActiveX control (now replaced by the managed Windows Forms control) deployed over the Internet as a Web page. This application is much like other client applications: it is executed natively, has access to local resources, and includes graphical elements.

In the past, developers created such applications using $\mathrm{C} / \mathrm{C}++$ in conjunction with the Microsoft Foundation Classes (MFC) or with a rapid application development (RAD) environment such as Microsoft ${ }^{\circledR}$ Visual Basic ${ }^{\circledR}$. The .NET Framework incorporates aspects of these existing products into a single, consistent development environment that drastically simplifies the development of client applications.

The Windows Forms classes contained in the .NET Framework are designed to be used for GUI development. You can easily create command windows, buttons, menus, toolbars, and other screen elements with the flexibility necessary to accommodate shifting business needs.

\section{Project Description}

This web-based application is for the employees of an organization for processing their visa applications. HR Manager is considering as Site Admin and he is having the control over all the users. Administrator can create any no of Customer logins and authenticated them. Hr Executives are the responsible persons to execute the process of Visa applications and their Onsite Details. Company Employees, those who are willing enquire about visa status are able to login into this portal. Their empID is considered as username and their password will be given by Company HR during his/her joining. Onsite employees may apply for visa renewal through this project.

- $\quad$ Replace Manual Processing system with an automated one.

- Speedup Transactions

- Reduce the chances of malpractices associated in a manual system.

- Reduce the workload involved in processing

- Update information system and provide easy access to corresponding information.

- Fully automated data storing through online

- Online Checking of Visa Status of employee

\section{Visa Processing System}

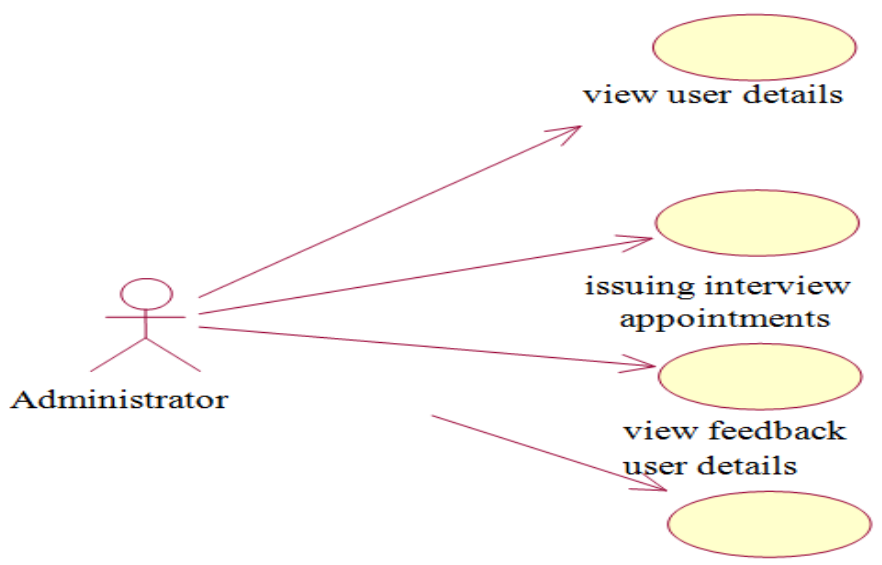

cancel appointments 


\section{Modules}

To achieve the above target, our project Visa Processing System will be categorized as the following Modules.

- Admin Module

- Visa Processing Module

- Onsite Communication Module

- Reporting Module

\section{Module Description:}

\section{Admin Module}

Employee Authentication will be finalized by the employee main database. Admin can view the visa renewal information, visa status information. He can generate all possible reports like interview reports, results report .He is having the control over the all the users. Hr Manager will describe the utilities assessed by an Customer. He can view the application entries, enquiry details, interview details, result details, onsite departure details, and onsite arrival details.

\section{Visa Processing Module:}

This is a main module. It handles all the details regarding visa application, submitted documents, employee particulars, passport details, earlier onsite visits and the details regarding their earlier tour details. This will be entered by. After applying visa every applicant should attain enquiry and interviews held by consulates. These enquiry and interview details are stored in this module. The result also logged in this module. These details also entered by Customer.

\section{Onsite Data Module:}

Candidates onsite particulars, like visa issuing date, flight date, no of days spending at abroad, returning date, onsite job particulars are stored through this module. Customer's onsite progress like project name, working period, working place and etc., are entered by the Customer itself. In the case of renewal of visa, Customers can give a request to the Administrator for their renewal of visa. These requests are viewed by Administrator and have process on for Visa Renewal. Employees can view the visa renewal information and visa process status like current visa status and finished visa status for different countries.

\section{Conclusion}

The Visa Processing Information System is a web-based application for tracking the visa transactions in a visa processing company which provide customized solutions to meet company/customer needs. This application software has been computed successfully and was also tested successfully by taking "test cases". It is user friendly, and has required options, which can be utilized by the user to perform the desired operations.

The software is developed using Java as front end and MySQL as back end in Windows environment. The goals that are achieved by the software are:

- Instant access.

- Improved productivity.

- Optimum utilization of resources.

- Efficient management of records.

- Simplification of the operations.

- Less processing time and getting required information.

- User friendly.

- Portable and flexible for further enhancement.

[1]. Ramandeep Kaur-Aman Arora, "Configuration Management: A Review" International Journal of Advanced Research in Computer Science and Software Engineering Volume 2013

[2]. Mark Lindeman, Philip B.Stark, "A Gentle Introduction to Risk -limiting Audits" IEEE 2012

[3]. Yongchang Ren, Tao Xing, Qiang Quan, "Software Configuration Management of Version Control Study Based on Baseline" IEEE 2010

[4]. Mark Staples,Paul L Bannerman,"Software Configuration Management in Global Software Development: A Systematic Map” IEEE 2010

[5]. Tao Xing "Software Configuration Management of Change Control Study Based on Baseline" IEEE 2010

[6]. Bo Yang, "Knowledge-Enhanced Change Audit for Configuration Management" IEEE 2010

[7]. Steven M.Bellovin,Randy Bush "Configuration Management and Security" IEEE 2009 